THE Astrophysical Journal, 498:773-778, 1998 May 10

(C) 1998. The American Astronomical Society. All rights reserved. Printed in U.S.A.

\title{
THE CASE FOR RARITY OF CHONDRULES AND CALCIUM-ALUMINUM-RICH INCLUSIONS IN THE EARLY SOLAR SYSTEM AND SOME IMPLICATIONS FOR ASTROPHYSICAL MODELS
}

\author{
DEREK W. G. SEARS \\ Cosmochemistry Group, Department of Chemistry and Biochemistry, University of Arkansas, Fayetteville, AR 72701 \\ Received 1997 July 16; accepted 1997 December 8
}

\begin{abstract}
The high-temperature materials in chondritic meteorites, the chondrules (silicate spherules produced by flash heating) and refractory inclusions (the CAI, or calcium-aluminum-rich inclusions), have been used to place major constraints on astrophysical models for the early solar system. Among the meteorites falling to Earth, the CAI are rare, being only $15 \%$ by volume of a class that constitutes $0.84 \%$ of observed falls, however chondrules are ubiquitous. Here it is pointed out $\sim 96 \%$ of the meteorites entering the atmosphere contain few or no chondrules because atmospheric passage destroys such meteorites 1000 times more effectively than the tougher chondrule-rich material. Furthermore, the mechanisms for transferring meteorites from the asteroid belt to Earth do not sample the belt representatively, so that even the $4 \%$ of chondrule-rich material entering the atmosphere may be an overestimate. Most asteroids have surfaces resembling meteorite classes that are chondrule-poor or chondrule-free. Foreign clasts in meteorites, which are an independent sampling of asteroidal material, usually resemble chondrule-poor or chondrule-free carbonaceous chondrites. Cosmic-ray ages and gas-retention ages for meteorites indicate that most stony meteorites are coming from just a few asteroids. A strong case can thus be made that chondrules and CAI were rare in the inner solar system during planet and planetesimal formation and that they do not provide a strong constraint on astrophysical ideas for the evolution of the early solar nebula.
\end{abstract}

Subject headings: dust, extinction — meteors, meteoroids — solar system: formation

\section{INTRODUCTION}

Two of the best-studied components in chondritic meteorites are the chondrules (King 1983; Hewins, Jones, \& Scott 1996) and refractory inclusions (calcium-aluminumrich inclusions, CAI; MacPherson et al. 1988), but, as the large number of proposed processes for the formation of chondrules suggests (Boss 1996), much of their origin and history is controversial. Nevertheless, it seems clear that they were formed in high-temperature events of relatively short duration early in the history of the solar system.

Despite the uncertainty over their formation, the suggestion is often made that chondrules and CAI place major constraints on astrophysical processes in the early solar nebula. For example Levy (1988), in discussing the energetics of chondrule formation, suggests that the abundance of chondrules requires that the amount of energy involved in chondrule formation was comparable to the total gravitational energy available in the nebula at their formation location, widely assumed to be the asteroid belt. Wood (1985) has used this notion to argue that chondrules did not form during the late relatively quiescent phases of nebular evolution but during the gravitational collapse of the nebula. Most recently, Shu, Shang, \& Lee (1996) have argued that CAI and chondrules travelled large distances across the nebula so that the energy source could have been that producing the bipolar outflows characteristic of young stars.

However, it is possible to argue that chondrules and CAI are rare, requiring special circumstances for their formation, and that the total energy involved in their formation was small relative to nebula energetics. Chondrules and CAI have attracted considerable interest partly because of their interesting properties and partly because, in the case of the chondrules especially, of a number of observational biases that give a false impression of abundance.

\section{OBSERVATIONAL BIASES}

The widespread perception that chondrules are important constituents of chondrites reflects the large numbers of known chondrule-rich ordinary chondrites (the $\mathrm{H}, \mathrm{L}$, and LL chondrites; Table 1). The chondrule abundance in enstatite (EH and EL) is somewhat smaller but comparable to that of the volatile-poor carbonaceous (CV and $\mathrm{CO}$ ) chondrites, while the volatile-rich carbonaceous (CI and $\mathrm{CM}$ ) chondrites contain few chondrules, if any. The CI chondrites are the chondrite class closest to solar in composition and are nearly $20 \%$ by volume of water (Jarosewich 1991). The CAI are even rarer, constituting only $15 \%$ by volume of the relatively rare CV chondrite class $(0.84 \%$ of observed falls), and only traces in other classes. Thus only $\sim 0.1 \%$ by volume of primitive solar system material falling to Earth consists of CAI. The igneous HEDM meteorites (howardites-eucrites-diogenitesmesosiderites) and the iron meteorites contain only trace abundances of objects identical in structure to chondritic chondrules (Olsen \& Jarosewich 1971; Olsen et al. 1990).

The first bias is that the atmosphere screens all but the most robust meteorites from falling to Earth. The CI and CM chondrites are actually friable "mud balls." Their fragment mass decreases by a factor of $10^{3}$ as they pass through the atmosphere (Fig. 1; Baldwin \& Shaeffer 1971). (Baldwin \& Shaeffer actually refer to "carbonaceous chondrites," but the parameters they used apply to CI and CM chondrites.) In contrast, ordinary chondrites are extremely tough, dry coherent rocks that suffer little destruction as they pass through the atmosphere. The likelihood of recovery of a meteorite on Earth is proportional to its final mass, so that fall statistics should be adjusted by multiplying the number of carbonaceous meteorite falls by 1000 . After such adjustment, $96.5 \%$ of observed falls would be CI and CM chondrite, rather than the observed $2.26 \%$, and if this were 
TABLE 1

Major and Important Meteorite Classes, Their Abundance (IN Terms of Fall Rate on Earth), and Chondrule Concentration. Asteroid Classes and Their Relative Abundance

\begin{tabular}{|c|c|c|c|}
\hline Class $^{\mathrm{a}}$ & $\begin{array}{c}\text { Abundance } \\
\text { (percent) }\end{array}$ & $\underset{\text { (percent) }}{\text { Chondrule Abundance }}{ }^{\mathrm{c}}$ & Meteorite Match \\
\hline \multicolumn{4}{|l|}{ Meteorites: } \\
\hline $\mathrm{H}, \mathrm{L}, \mathrm{LL}$. & 79.5 & $65-75$ & \\
\hline $\mathrm{EH}, \mathrm{EL} \ldots \ldots \ldots$ & 1.56 & $20-40$ & \\
\hline $\mathrm{CO} \ldots \ldots \ldots \ldots$ & 0.60 & $35-40$ & \\
\hline $\mathrm{CV} \ldots \ldots \ldots \ldots \ldots$ & 0.84 & $35-45$ & \\
\hline $\mathrm{CM} \ldots \ldots \ldots \ldots$ & 2.2 & $\sim 9$ & \\
\hline $\mathrm{CI} \ldots \ldots \ldots \ldots \ldots$ & 0.06 & 0 & \\
\hline HED ............. & 7.02 & $\sim 0$ & \\
\hline Irons $\ldots . . . \ldots \ldots$ & 4.95 & $\sim 0$ & \\
\hline \multicolumn{4}{|l|}{ Asteroids: } \\
\hline D ....... & 6.2 & & IDP \\
\hline$P \ldots \ldots \ldots \ldots \ldots$ & 4.3 & & IDP \\
\hline $\mathrm{C} \ldots \ldots \ldots \ldots \ldots \ldots$ & 22.8 & & $\mathrm{CI}, \mathrm{CM}$ \\
\hline $\mathrm{T} \ldots \ldots \ldots \ldots \ldots$ & 1.3 & & $\operatorname{Ir}(\mathbf{S})$ \\
\hline$B+G+F \ldots \ldots$ & 9.1 & & CI, CM \\
\hline $\mathrm{Q} \ldots \ldots \ldots \ldots \ldots \ldots$ & 0.41 & & $\mathrm{H}, \mathrm{L}, \mathrm{LL}$ \\
\hline $\mathrm{V} \ldots \ldots \ldots \ldots \ldots$ & 0.41 & & HED \\
\hline $\mathrm{R} \ldots \ldots \ldots \ldots \ldots \ldots$ & 0.10 & & $?$ \\
\hline $\mathrm{S}(\mathrm{IV}) \ldots \ldots \ldots \ldots \ldots$ & 11.0 & & Lod, Win, OC, irons \\
\hline S(others) ........ & 25.9 & & Pal, Ure, Bra, Lod, Mes, Win, Ste \\
\hline $\mathrm{A} \ldots \ldots \ldots \ldots \ldots \ldots$ & 0.71 & & Pal \\
\hline$M \ldots \ldots \ldots \ldots \ldots$ & 4.3 & & Irons, EC \\
\hline $\mathrm{E} \ldots \ldots \ldots \ldots \ldots$ & 1.3 & & Aub \\
\hline
\end{tabular}

${ }^{a}$ For asteroid class and abundance, see Tholen (1989) and Gaffey et al. (1993a).

${ }^{\mathrm{b}}$ For meteorite abundance, see Sears \& Dodd (1988).

c For meteorite chondrule abundance, see Grossman (1988) and Zhang et al. (1996).

${ }^{d}$ Meteorite classes most closely resembling the asteroid class (Gaffey et al. 1993b). IDP, interplanetary dust particles; $\operatorname{Ir}(\mathrm{S})$, troilite-rich iron meteorites; OC, ordinary (H, L, LL) chondrites; Lod, Iodranites; Win, winonites; Pal, pallasites; EC, enstatite (EH and EL) chondrites; Aub, aubrites; Ure, ureilites; Bra, brachinites; Ste, Steinbach; Mes, mesosiderites; HED, howardites-eucrites-diogenites. All but OC, EC, HED, IDP, and irons are rare igneous meteorites.

representative of the asteroid belt, then chondrules are an extremely rare type of primordial material.

The second bias that suggests chondrules might be more abundant in the early solar system than they actually were concerns the mechanism by which meteorites were transferred from the asteroid belt to Earth. There are two views on how meteorites reach Earth; one involves stochastic collisions, and the other involves evolution through orbital resonances (Greenburg \& Nolan 1979). It is doubtful that either mechanism samples the asteroid belt representatively. In the case of collisions, the nature of the flux of meteorites to Earth depends on the nature of the impacting objects. Delivery of meteorites to Earth without impact assumes that the objects are accelerated to high eccentricity orbits by being close to orbital resonances with Jupiter or Saturn so that the resonances may be regarded as "escape hatches." Only a small proportion of the asteroids have orbits close to resonances (Fig. 2). In fact, Farinella, Froeschle, \& Gonczi (1993), Morbodelli et al. (1994), and Gaffey \& Gilbert (1996) argue that a single $\mathrm{S}(\mathrm{IV})$ asteroid orbiting near both the $v_{6}$ resonance with Saturn and the 1:3 resonance with Jupiter, 6 Hebe (which arguably also has the appropriate reflectivity spectrum), is the sole source of the major $\mathrm{H}$ chondrite meteorite class.

Of course it is possible that the actual mechanism for transferring objects from the asteroid belt to Earth is a combination of these two processes, and that impacts are more likely near resonances, or that impacts are required to inject asteroids into the resonances.

There is considerable evidence in the meteorite data that some of the larger classes are coming from single-source objects. The remarkable uniformity of compositional, isotopic, and textural properties is circumstantial evidence, but the clustering of cosmic-ray exposure ages or $\mathrm{K}-\mathrm{Ar}$ ages is very strong evidence for solitary parent bodies for the ordinary chondrite classes. The duration of exposure to cosmic rays can be deduced from the abundance of stable cosmicray-produced nuclides in the meteorite, like ${ }^{3} \mathrm{He}$ or ${ }^{21} \mathrm{Ne}$, and histograms of cosmic-ray exposure ages often contain peaks suggesting that most members of that class were once part of a single parent object that was disrupted by a single major impact. Cosmic-ray exposure ages for iron meteorites tend to be $10^{8}-10^{9} \mathrm{yr}$, but for stony meteorites, the ages are quite short $\left(\sim 10^{7} \mathrm{yr}\right.$; Fig. 3$)$. The best known example of tight clustering in these ages is the 8 Ma peak displayed by the $\mathrm{H}$ chondrites, but the LL chondrites show a 17 Ma peak and $\mathrm{L}$ chondrites have similar but less marked clusters of ages (Marti \& Graf 1992). Other chronometers involving gaseous daughter products that would be readily lost during impact heating produce similar results. The K-Ar ages for the L chondrites show two clusters, one corresponding to about $4.6 \mathrm{Ga}$, the formation age for the solar system, and another to about $500 \mathrm{Ma}$. Meteorites in the 500 Ma age peak often contain petrographic evidence for a shock heating event, suggesting that most of the L chondrites were part of a single parent object that violently broke up $500 \mathrm{Ma}$ ago (Bogard 1995; Fig. 3). There is also evidence that the $\mathrm{H}$ chondrite class is being freshly liberated from a single fairly localized source. Dates of fall tend to cluster (Dodd, Wolf, \& Lipschutz 1993) and the induced thermoluminescence properties and metallographic cooling rates of $\mathrm{H}$ chondrites seem to vary with terrestrial age (time 


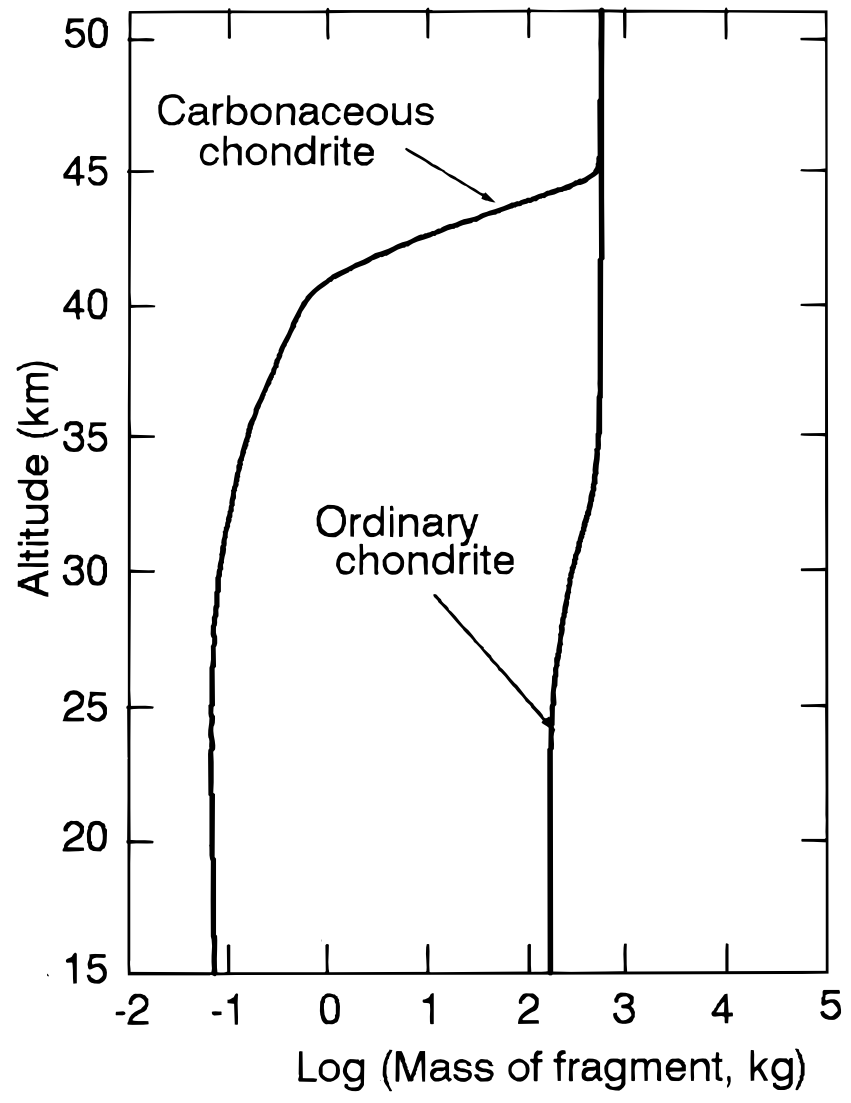

FIG. 1.-Plot of fragment mass as a function of height as meteorites pass through the atmosphere. Weak, friable meteorites like the CI and CM chondrites undergo considerable fragmentation as they pass through the atmosphere, whereas the relatively tough ordinary chondrites do not. Since the chances of recovery scale directly with surviving mass, the atmosphere reduces by a factor of 1000 the apparent flux of CI and CM chondrites in the vicinity of Earth (after Baldwin \& Shaeffer 1971).

since fall to Earth; Benoit \& Sears 1996).

\section{EVIDENCE FOR BIAS?}

On the basis of the spectra of sunlight reflected from their surfaces, asteroids have been sorted into a number of classes, some of which have matches among the meteorites reaching Earth (Table 1). The primitive D, $\mathrm{P}$, and $\mathrm{C}$ classes have spectra that suggest clays and organics; the large $\mathrm{C}$ class has been likened to $\mathrm{CI}$ and $\mathrm{CM}$ chondrites; while the $\mathrm{T},(\mathrm{B}+\mathrm{G}+\mathrm{F})$, and $\mathrm{Q}$ classes appear to be opaque mineral-bearing clays and perhaps metamorphosed objects. The spectra of $Q$ asteroids provide the only really good match for ordinary chondrites, but they are very rare $(0.41 \%)$. Vesta and the other V-type asteroids have spectra that match the nonchondritic HEDM meteorites (McCord, Adams, \& Johnson 1973). The $\mathrm{S}$ class is often likened to the ordinary chondrites on the assumption that some alteration process has camouflaged their surfaces. However, the $\mathrm{S}$ class is extremely diverse. It has been divided into seven smaller groups and only the S(IV) class can reasonably be related to the ordinary chondrites (Gaffey et al. 1993a). Even the S(IV) class requires that some process has modified its surface to redden its spectrum (Chapman 1996). The $\mathrm{R}, \mathrm{A}, \mathrm{N}, \mathrm{E}$, and remaining $\mathrm{S}$ classes resemble various groups of igneous stony meteorites, the iron meteorites, and stonyiron meteorites (Gaffey et al. 1993b). Thus, less than $11 \%$ of

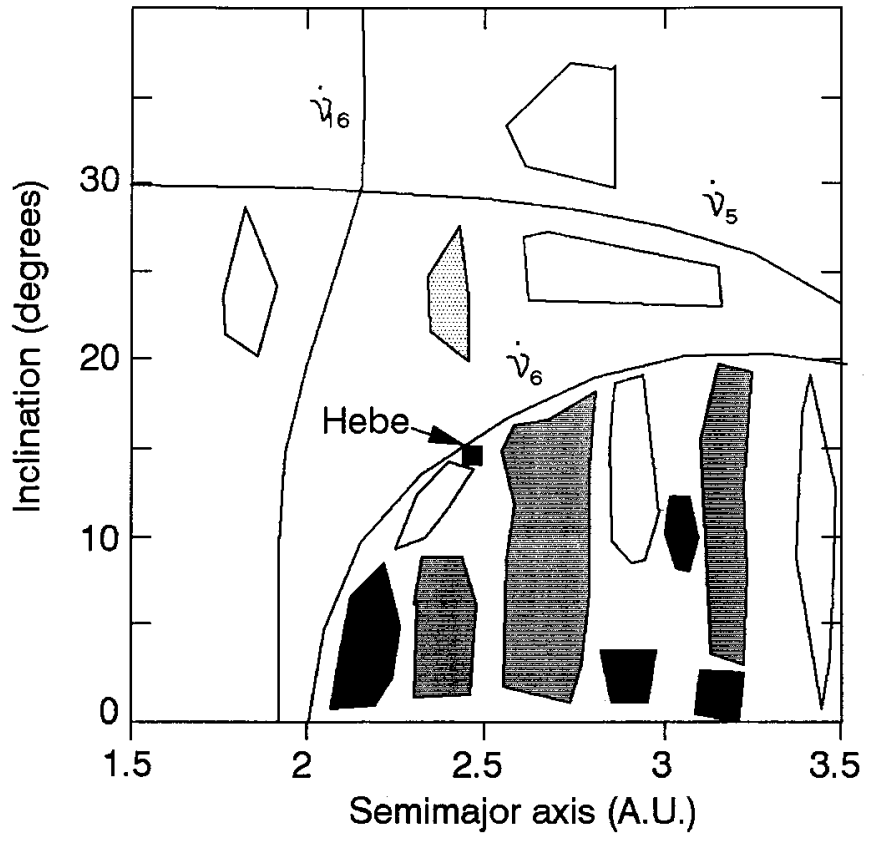

FIG. 2.-Plot of inclination against eccentricity for asteroid orbits illustrating the large number of possible asteroid orbits and the location of the resonances that enable asteroid fragments to leave the asteroid belt and eventually find their way to Earth. Asteroid 6 Hebe lies near two resonances $\left(v_{6}\right.$ and $\left.3: 1\right)$ and may be the source of the $\mathrm{H}$ chondrites. The density of the shading is a qualitative reflection of the asteroid density in that region of inclination-semimajor axis space. (Versions of this figure showing individual data can be found, for instance, in Binzel et al. 1989).

the asteroids have spectra that resemble the ordinary chondrites, and the number may be very much less than $11 \%$.

A second line of evidence that most of the small objects in the inner solar system are not ordinary chondrite like is provided by the foreign clasts (rock fragments) found in meteorites regardless of class. Impact onto the surface of an Earth-bound meteorite, followed by incorporation into the fabric of the host meteorite by the usual lithification processes, is a means of bringing to Earth meteorites that might not otherwise survive atmospheric passage. Despite alteration during impact or subsequently, 18 of 22 foreign clasts found in meteorites and listed by Wilkening (1976) were carbonaceous (Table 2). Most are CM-like. Without giving similarly complete data, Anders (1978) subsequently found that $75 \%$ of foreign clasts in meteorites were carbonaceous, and Lipschutz, Gaffey, \& Pellas (1989) found that greater than $60 \%$ are carbonaceous. Even allowing for the statistics of small numbers, alteration during emplacement, and the difficulty of classifying these sometimes small clasts, the abundance of carbonaceous clasts is clearly 30-35 times higher than the $2.26 \%$ abundance of carbonaceous chondrite falls.

\section{IMPLICATIONS}

It can thus be argued that chondrules and CAI were probably rare in the early solar system. Earth's atmosphere heavily biases terrestrial meteorite collections toward chondrule-rich meteorites, virtually all asteroids have surfaces similar to chondrule-poor or chondrule-free meteorite classes, foreign clasts being brought to Earth by tougher meteorites are predominantly of chondrule-poor or chondrule-free classes, and the meteorite data seem to indicate that the chondrule-rich meteorites are coming from 

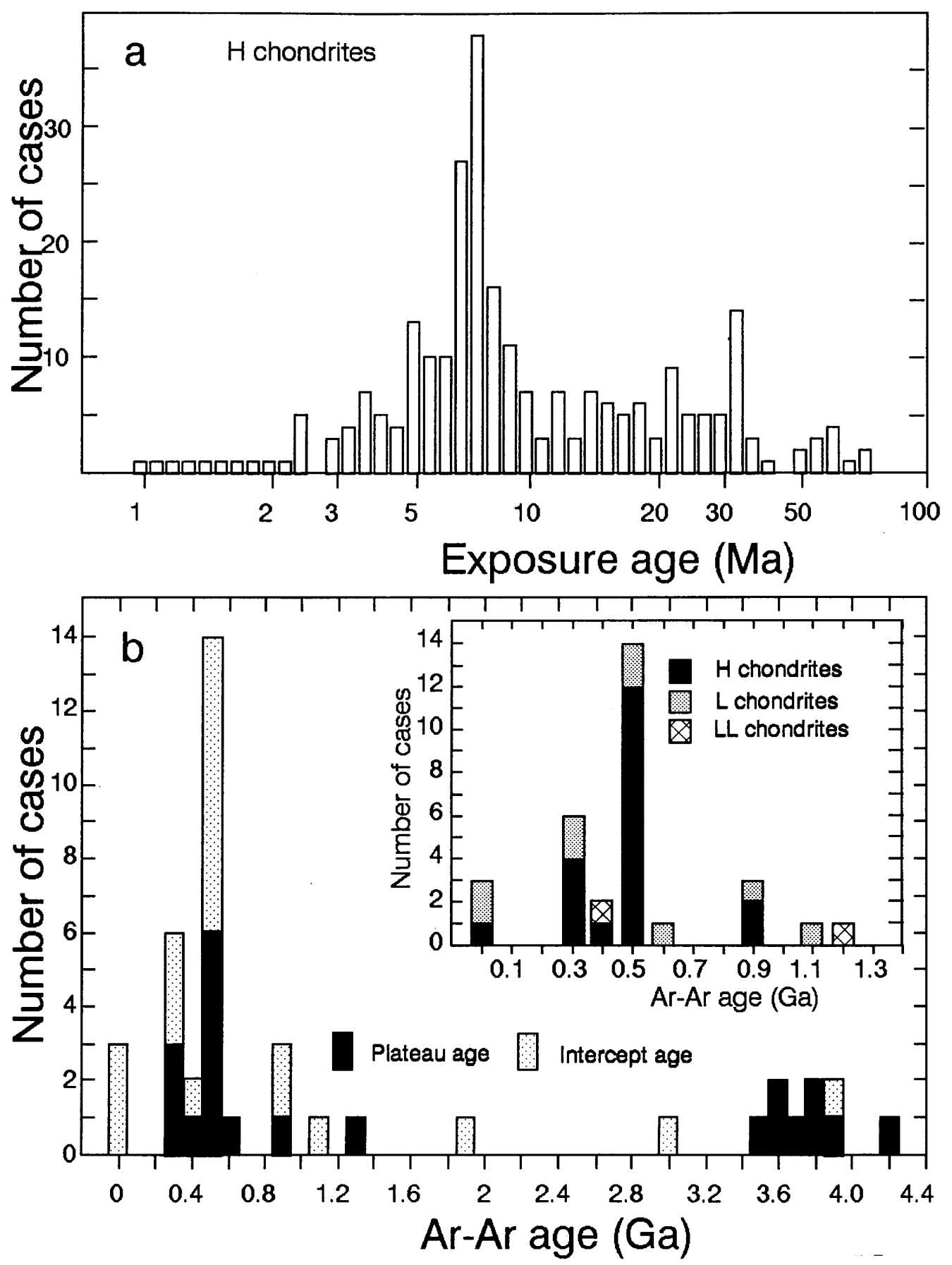

Fig. 3. - (a) Histogram of cosmic-ray exposure ages for $\mathrm{H}$ chondrites. The strong peak at $8 \mathrm{Ma}$ indicates that a large number of members of this class broke up at the time, presumably as a result of a single large impact. (b) Ar-Ar ages for L chondrites show a peak at 500 Ma containing meteorites that show petrographic evidence for an intense shock-heating event. These data indicate that (1) impacts are important in the history of meteorites and (2) most members of these two major chondrite classes were part of a single body prior to these break-ups (after Marti \& Graf 1992 and Bogard 1995).

very few parent asteroids. They were the asteroids that were either fortuitously close to a resonance or happened to undergo a major impact necessary to send fragments to Earth; perhaps both impacts and resonances were involved. It is not necessary to invoke nebula-wide mechanisms involving nebula-scale energy input to account for the chondrules or the CAI. They may be the result of highly localized processes in which the amounts of energy involved are large only on a highly localized basis.

Detailed objections can be made to some of the nebulascale models for chondrule formation. For example, chondrules formed several million years after the first-formed solar system solids and probably after the nebula had dissipated, oxygen, sulfur, and sodium fugacities were several orders of magnitude higher than any independent astrophysical model would predict, chondrules cooled many orders of magnitude more slowly than would be expected for a nebular environment, and chondrule densities in certain classes are much higher than can readily be explained by various nebular theories (Sears et al. 1995). If CAI travelled nebula-scale distances, as suggested by Shu et al. (1996), why are they only abundant in a small, rare class of chondrites and not size sorted with respect to chondrules in other classes? 
TABLE 2

Foreign Clasts In METEORITES ${ }^{\mathrm{a}}$

\begin{tabular}{|c|c|c|}
\hline Host Chondrite & Host Class & Clast Class \\
\hline Abbott & H5 & CM \\
\hline Allende ............ & CV3 & $\mathrm{CM}$ \\
\hline Bencubbin..$\ldots \ldots \ldots \ldots$ & Chon & $\mathrm{LL}, \mathrm{CM}, \mathrm{ch}$ \\
\hline Cumberland Falls ....... & Aub & chon \\
\hline Cynthiana $\ldots \ldots \ldots \ldots \ldots$ & L4 & $\mathrm{C}$ \\
\hline Dimmitt........ & $\mathrm{H} 3,4$ & $\mathrm{C}$ \\
\hline Holyhoke...$\ldots \ldots \ldots$ & $\mathrm{H} 4$ & $\mathrm{C}$ \\
\hline Jodzie...$\ldots \ldots$ & LL3 & $\mathrm{CM}$ \\
\hline Kapoeta ................ & How & $\mathrm{CM}$ \\
\hline Krymka $\ldots \ldots \ldots \ldots \ldots$ & LL3 & $\mathrm{CM}$ \\
\hline Lance........... & $\mathrm{CO} 3$ & $\mathrm{CM}$ \\
\hline Leoville ....... & CV3 & $\mathrm{CM}$ \\
\hline Murchison .... & CM2 & $\mathrm{C}$ \\
\hline Plainview $\ldots \ldots \ldots \ldots \ldots$ & H5 & $\mathrm{CM}$ \\
\hline Pultusk ........ & H5 & $\mathrm{C}$ \\
\hline Rio Negro ...... & L3 & $\mathrm{C}$ \\
\hline St. Mesmin $\ldots \ldots \ldots \ldots \ldots$ & LL6 & $\mathrm{H}$ \\
\hline Sharps .................. & $\mathrm{H} 3$ & CM \\
\hline Tieschitz $\ldots \ldots \ldots \ldots \ldots$ & H3 & $\mathrm{C}$ \\
\hline Tysnes Island.. & H4 & $\mathrm{C}$ \\
\hline Weatherford ............. & Chon & chon \\
\hline Weston ................. & $\mathrm{H} 4$ & $\mathrm{C}$ \\
\hline
\end{tabular}

${ }^{\text {a }}$ After Wilkening (1976) with minor updates.

The strongest argument in favor of forming chondrules in the nebula is a default argument; formation by impact onto an asteroidal surface was thought unlikely because impact velocities were thought insufficient and that impact ejecta would be lost from the asteroid. It is now known that neither of these conclusions is correct. Mariner and Galileo spacecraft images of the Martian satellites and asteroids Gaspra and Ida indicate that asteroids have thick dusty surface regoliths, and ejecta is not lost from even the smallest asteroids. We also now know that by the time the chondrules formed, several million years after the asteroids, Jupiter had increased relative velocities to $5 \mathrm{~km} \mathrm{~s}^{-1}$, sufficient to produce impact melt spherules in a dusty target. In fact, it seems unlikely that the nebula still existed at the time chondrules formed, several million years after the CAI formed. There is a strong consensus that the asteroids and planets formed rapidly (a few times $10^{5} \mathrm{yr}$; see, e.g., Cameron 1995), rather than the $10^{7} \mathrm{yr}$ or more of $\tau$ Tauri stars and certain circumstellar nebulae (Podosek \& Cassen 1994).

A more probable scenario for chondrule formation is by impact into an asteroidal regolith. Objects identical in size and texture to meteoritic chondrules are found in certain lunar breccias. The size and abundance of chondrules in the Apollo 14 breccias is very similar to those of the CM chondrites (Sears et al. 1995; Symes et al. 1998). The size of the Moon precludes the long flight times for most impact ejecta required by estimated chondrule cooling times. Only an impact the size of Imbrium can produce fully crystalline impact spherules on the Moon. Other impacts produce agglutinates or glassy impact spherules commonplace on the Moon. In contrast, most impacts on asteroids will produce crystalline spherules (i.e., chondrules), and agglutinates and glassy spherules will be rare or absent. Oxygen isotope systematics of meteoritic chondrules are also more consistent with an origin by impact into a regolith, especially one on a CI-like asteroid with many volatiles (as we have proposed). All the proposed mechanisms for oxygen isotope manipulation could occur in the regolith: heterogeneity in the precursors, mass-independent fractionation of the sort described by Thiemens (1996) exchange with gases or solids, mixing between gases or solids, massdependent reactions between gas and chondrules, and mass-dependent evaporation and recondensation. At least three reservoirs of oxygen were present (interstellar grains, chemically combined water, or water-rich gases mobilized from the interior), and their compositions might have varied with time. It is difficult to imagine that these processes could occur in the nebula, where gas and particle densities were low and mixing lengths large.

The only argument against chondrules being a surface phenomenon are the slow cooling rates inferred from the composition and dimensions of metal grains in meteorites. However, given that asteroids have thick regoliths, it no longer seems necessary to invoke deep burial to produce the slow cooling rates. Burial in a few kilometer-thick regolith is all that is required (Akridge, Benoit, \& Sears 1997a, 1997b).

Regardless of these details, the fundamental premise of many astrophysical models for chondrule formation and the early solar system can be reasonably challenged. There is no reason to suppose that chondrule or CAI formation was widespread and common in the early solar system, and astrophysical models constrained to produce frequent heat pulses are not necessary. They were probably produced by impact on asteroids the fragments of which are overrepresented in our terrestrial collections. Similar arguments to these have been made in connection with the interpretation asteroid spectra using meteorite data (Bell 1995).

\section{REFERENCES}

Akridge, G., Benoit, P. H., \& Sears, D. W. G. 1997a, Lunar Planet. Sci., 27, 13

1997b, Icarus, in press

Anders, E. 1978, NASA Conf. Pub., 2053, 57

Baldwin, B., \& Shaeffer, Y. 1971, J. Geophys. Res., 76, 4653

Bell, J. F. 1995, Meteoritics, 30, 484

Benoit, P. H., \& Sears, D. W. G. 1996, MAPS, 21, 81

Binzel, R. P., Gehrels, T., \& Matthews, M. S., ed. 1989, Asteroids, Vol. 2 (Tucson: Univ. Arizona Press)

Bogard, D. D. 1995, Meteoritics, 30, 244

Boss, A. P. 1996, in Chondrules and the Protoplanetary Disk, ed. R. H. Hewins, R. H. Jones, \& E. R. D. Scott (Cambridge: Cambridge Univ. Press), 257

Cameron, A. G. W. 1995, Meteoritics, 30, 133

Chapman, C. R. 1996, MAPS, 31, 699

Dodd, R. T., Wolf, S. F., \& Lipschutz, M. E. 1993, J. Geophys. Res. E, 98, 15,105

Farinella, P., Froeschle, C., \& Gonczi, R. 1993, Celest. Mech. Dyn. Astron., 56,287

Gaffey, M. J., Bell, J. F., Brown, R. H., Burbine, T. H., Piatek, J. L., Reed,

K., \& Chaky, D. A. 1993a, Icarus, 106, 573

Gaffey, M. J., Burbine, T. H., \& Binzel, R. P. 1993b, Meteoritics, 28, 161 Gaffey, M. J., \& Gilbert, S. L. 1996, MAPS, in press

Greenberg, R., \& Nolan, M. C. 1979, in Asteroids, Vol. 2, ed. R. P. Binzel, T. Gehrels, \& M. S. Matthews (Tucson: Univ. Arizona Press), 778

Grossman, J. N. 1988, in Meteorites and the Origin of the Solar System, ed.

J. F. Kerridge \& M. S. Matthews (Tucson: Univ. of Arizona Press), 619

Hewins, R. H., Jones, R. H., \& Scott, E. R. D., ed. 1996, Chondrules and the Protoplanetary Disk (Cambridge: Cambridge Univ. Press)

Jarosewich, E. 1991, Meteoritics, 25, 323

King, A. E. 1983, Chondrules and their Origins (Houston: Lunar Planetary Institute)

Levy, E. H. 1988, in Meteorites and the Origin of the Solar System, ed. J. F. Kerridge \& M. S. Matthews (Tucson: Univ. of Arizona Press), 697

Lipschutz, M. E., Gaffey, M. J., \& Pellas, P. 1989, in Asteroids, Vol. 2, ed. R. P. Binzel, T. Gehrels, \& M. S. Matthews (Tucson: Univ. Arizona Press), 740

MacPherson, G. J., et al. 1988, in Meteorites and the Origin of the Solar System, ed. J. F. Kerridge \& M. S. Matthews (Tucson: Univ. Arizona Press), 746

Marti, K., \& Graf, T. 1992, Ann. Rev. Earth Planet. Sci., 20, 221

McCord, T. B., Adams, J. B., \& Johnson, T. V. 1973, Science, 168, 1445 
Morbedelli, M., Gonczi, R., Froeschle, C., \& Farinella, P. 1994, A\&A, 282, 955

Olsen, E., Fredriksson, K., Rajan, S., \& Noonan, A. 1990, Meteoritics, 25, 187

Olsen, E., \& Jarosewich, E. 1971, Science, 174, 583

Podosek, F. A., \& Cassen, P. 1994, Meteoritics, 29, 6

Sears, D. W. G., \& Dodd, R. T. 1988, in Meteorites and the Origin of the Solar System, ed. J. F. Kerridge \& M. S. Matthews (Tucson: Univ. of Arizona Press), 3

Sears, D. W. G., Huang, S., \& Benoit, P. H. 1995, Lunar Planet. Sci., 26, 1263

Shu, F. H., Shang, H., \& Lee, T. 1996, Science, 271, 1645
Symes, S. J. K., Taunton, A., Benoit, P. H., \& Sears, D. W. G. 1998, MAPS, 33,13

Thiemens, M. H. 1996, in Chondrules and the Protoplanetary Disk, ed. R. H. Hewins, R. H. Jones, \& E. R. D. Scott (Cambridge: Cambridge Univ. Press), 107

Tholen, D. J. 1989, Asteroids Vol. 2, ed. R. P. Binzel, T. Gehrels, \& M. S. Matthews (Tucson: Univ. of Ariz. Press), 1139

Wilkening, L. L. 1976, Proc. Lunar Sci. Conf. 7, ed. R. B. Merrill (New York: Pergammon), 3549

Wood, J. A. 1985, Lunar Planet. Sci., 26, 1517

Zhang, C., et al. 1996, J. Geophys. Res. E, 100, 9417 